\title{
Schwerpunkt: Wohin führt Transparenz in der Demokratie?
}

Transparenz hat Konjunktur. Der Begriff wird in vielen öffentlichen Debatten verwendet, auch ohne Vorliegen einer präzisen Definition oder der genauen Klärung der konkreten Anwendungs- bzw. Problempotenziale. Im Zusammenhang mit den Verhandlungen zum transatlantischen Freihandelsabkommen TTIP, den Tätigkeiten von Geheimdiensten oder dem Drängen auf die Veröffentlichung von Politikergehältern: immer wieder wird die Forderung nach einem Mehr an Transparenz und Informationen von Politik, Medien und zivilgesellschaftlichen Akteuren erhoben. Immer dann, wenn diesen Forderungen nicht nachgekommen wird, schlägt die Stunde der Whistleblower, die bis dato unter Verschluss gehaltene Informationen und Dokumente zu veröffentlichen und somit - aus oftmals ganz unterschiedlichen Gründen - Transparenz und Öffentlichkeit herzustellen suchen. WikiLeaks, die NSA-Affäre und die Panama Papers bilden dabei nur die prominente Spitze des Eisbergs.

Aber hat Transparenz stets nur positive Effekte? Oder ist sie nicht etwa in der Politik oftmals hinderlich bei politischen Entscheidungen? Ist es in manchen Politikbereichen nicht sogar notwendig, Transparenz einzuschränken, Informationen zurückzuhalten, einen Arkanbereich zu bewahren, um Strategie- und Entscheidungsfähigkeit zu garantieren?

Das folgende Forum beschäftigt sich vor diesem Hintergrund mit der Bedeutung, dem Stellenwert und ausgewählten Dimensionen von Transparenz in der Demokratie. Aus theoretischer Perspektive beschäftigen sich Frank Gadinger und Taylan Yildiz mit der Qualität und Wirkungskraft von Transparenzerzählungen, die existenzielle Konfliktkonstellationen in den Blick rücken und dabei normative Prämissen demokratischer Ordnungen verändern können. Mariella Bastian und Susanne Fengler analysieren aus Sicht der politischen Systemforschung, wie Medien - selbst zentrale Akteure bei der Herstellung von Transparenz und Öffentlichkeit - mittels Transparenz und Einblick in ihre Arbeit verloren gegangenes Vertrauen und Glaubwürdigkeit zurückerlangen können. Sabrina Zajak thematisiert schließlich aus internationaler Perspektive, wie - insbesondere multinationale - Unternehmen zunehmend mit Forderungen nach Transparenz und Demokratisierung konfrontiert werden und damit ein wachsendes Demokratiedefizit von Staaten kompensiert werden soll. 